\title{
Subject Index, Vol. 72, 1996
}

This index does not contain items of the reports of the Chromosome Mapping Workshops 1, 3,10 , and 16 which are published in this volume.

Animals

Callithrix (marmoset) 325,331

cattle $37,212,342$

Cercopithecus aethiops tantalus (monkey) 9

chimpanzee 60

Equus caballus (horse) 162

goat 342

lemur 50

Macaca sylvana (macaque) 9

Macropus eugenii (tammar wallaby) 197

molossid bat 26

mouse 46, 56

Pithecia pithecia (ape) 9

rat 1,83

sheep 212,342

Banded chromosome analysis bovine(R) 342 chimpanzee(DAPI) 60 giraffe $\phi$ DAPI G/Q) 310 goat(R) 342 horse(GTG, CBG) 162 human(DAPIQ) 69 human(DAPI) 60, 63, 159, 200, 217, 294, 299,

303,316,322,339 human(G) 164,205,297,319 human(Q-like) 95 human(R) 78, 86, 171, 175, $177,185,189,191$,

208,242 marmoset(C, G, RE/C) 325 marmoset(G, C G/C, NOR) 331 mollosid bat(G, C) 26 mouse(C) 46 mouse $\mathrm{G}, \mathrm{C}) 56$ okapi(DAPI G/Q) $310 \operatorname{rat}(\mathrm{DAPI}) 83 \operatorname{rat}(\mathrm{R}) 1$ sheep(R) 342

Cell lines

SCLC derived GLC20 225 Chromosome aberration

bovine sterility in XY females 183

centromeric shift 9

colcemid effect on sperm chromosomes 164

correction by gene transfectíon 194

rearrangements induced by 5 -azaC 219

del( $12 p)$ in hematopoietic malignancies 229 Chromosome mapping workshops

chromosome 1 report 113

chromosome 3 report 255

chromosome 10 report 99

chromosome 16 report 271 Comparative gene mapping

ape, macaque, monkey 9

cattle 212,342

chimpanzee 60 
giraffe, okapi 310

goat 342

lemur 50

rat 1,83

sheep 212,342

Embryogenesis

detection of aneuploidy 43,46 maternal age and delayed fertilization 46 sperm age and karyotype 56

Fluorescent in situ hybridization (FISH) and PRINS aneuploidy assessment 34 chromosome painting 50, 229 DAPI G-band and signal improvement 200 interphaseFISH 200,219

multicolor FISH 43, 53, 63,219, 229, 306, 339 telomere detection 310

Gene expression

negative control in mouse hybrids 12 paternal X chromosome inactivation in marsupials 197

Gene mapping

ape, macaque, monkey

RBI 9 cattle

SOD1 37

EEF2, GGTA1, PRCR, SOX2, ZNF 146, ZNF164 342 chimpanzee

CSN2 60 goat

EEF2, GGTA1, PRCR, SOX2, ZNF 146, ZNF 164342 human

AQP3 303

CKAP1 208

CLG4B 159

CSN2 60

D1S3309E 306

endogenous retrovirus-K1O sequence 40

ESTs on chromosome 1372

GATA4 217

GJA3.GJB2 185

GLCLR 254

GLI4 297

GUSB 53

HCLS 175

HTR5A 187

LLGL 78

MMP7 179

MSMB 177

MYCL1 189

MYF5, MYF6 250

NHP2L1 191

PPIL1 242

PPP3CA, PPP3CB, PPP3R1 236

PSAP 316

PTAFR 205

SOX20 246

TFF3 299 
TK1 319

TPM4 294

UBE21 86 mouse

Gja3, Gjb2 185 rat

5SrDNA 1

Papl, Pap2, Papi, Reg 83 sheep

EEF2, GGTA1, PRCR, SOX2, ZNF 146, ZNF164 342 Human disorders

central core disease of muscle 215 dermatofibrosarcoma protuberans 171 Fanconi anemia

194 hematopoietic malignancies 229 hereditary neuropathy with liability to

pressure palsies 20 incontinentia pigmenti 43 male infertility 155 malignant hyperthermia

215 neurofibromatosis, Madelung deformity 95 Smith-Magenis syndrome 78 Werner

syndrome 63 Wilms' tumor 306

Hybrids

hamster-sheep 212

human-rodent 40, 63, 72, 159, 179, 187, 225,

236,254,294,299,306,322 mouse (Lck+) $\times($ Lck-) 12

Interphase mapping

FISH signal resolution 200 Interspecific backcross mapping

tammar wallaby 197

Karyotype

marmoset 325, 331 molossid bat 26

Libraries

chromosome specific 50 fetal brain cDNA 208

Linkage

centromere markers chromosome 569 CLG4B and chromosome 20 markers 159 marsupial X chromosome 197 polymorphic marker ordering in 3p21 225

Meiosis

colcemid induced human sperm abnormalities 164

reduced fertility in horse 162

segregation in males heterozygous for $\mathrm{Rb}$ translocations 56 Microsatellites

centromere chromosome 569

chromosome 3 hybrid panel 90

deletion mapping in HNPP 20

polymorphic markers flanking RYR1 215

sheep chromosome 6212

Physical mapping 17q25 319

chromosome 3 STS 90 chromosome 13 EST's 72 RCA gene cluster at lq32 339 triplet repeat

sequences in 6p23 5 Werner syndrome and markers in 8p21-pl2 63 YACmapatl2q21 250

Repeats

expanded triplet 5

Sequence

rat5SrDNA 1

bovine microsatellite 212

human chromosome 5 centromere marker 69

human CKAP1 208

human LLGL 78 


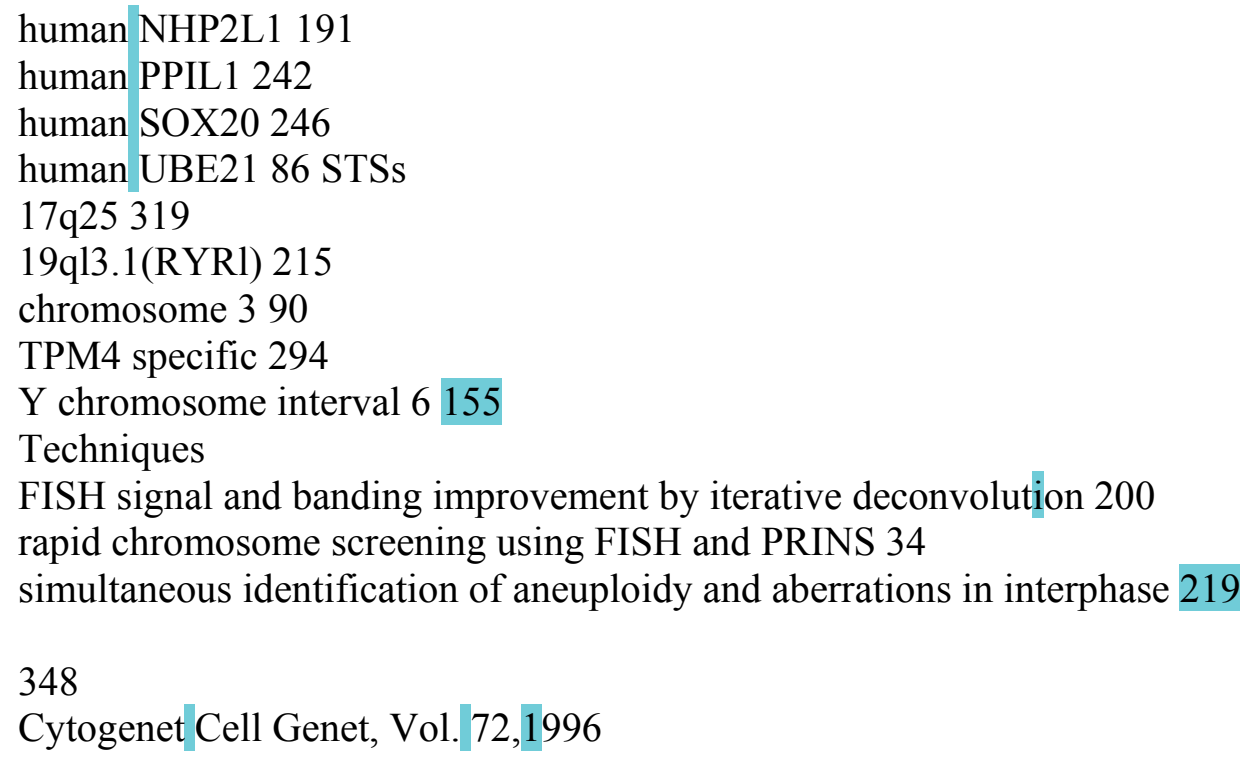

